\title{
Coupled Quintessence and Phantom Based On Dilaton
}

\author{
Z. G. HUANG \\ Department of Mathematics and Physics, \\ Huaihai Institute of Technology, 222005, Lianyungang, China \\ zghuang@hhit.edu.cn \\ H. Q. LU and W. FANG \\ Department of Physics, Shanghai University, Shanghai, China \\ alberthq_lu@staff.shu.edu.cn
}

\begin{abstract}
Based on dilatonic dark energy model, we consider two cases: dilaton field with positive kinetic energy(coupled quintessence) and with negative kinetic en$\operatorname{ergy}$ (phantom). In the two cases, we investigate the existence of attractor solutions which correspond to an equation of state parameter $\omega=-1$ and a cosmic density parameter $\Omega_{\sigma}=1$. We find that the coupled term between matter and dilaton can't affect the existence of attractor solutions. In the Mexican hat potential, the attractor behaviors, the evolution of state parameter $\omega$ and cosmic density parameter $\Omega$, are shown mathematically. Finally, we show the effect of coupling term on the evolution of $X\left(\frac{\sigma}{\sigma_{0}}\right)$ and $Y\left(\frac{\dot{\sigma}}{\sigma_{0}^{2}}\right)$ with respect to $N(\ln a)$ numerically.
\end{abstract}

Keywords: Dark energy; Dilaton; Quintessence; Attractor solution; Phantom

PACS numbers: $98.80 . \mathrm{Cq}$

\section{Introduction}

Observational data from SNe Ia[1], WMAP[2], SDSS[3], show that we are living in a spatially flat universe which consists of about two thirds dark energy with negative pressure, one third dust matter including cold dark matters plus baryons, and negligible radiation, and that our universe is undergoing an accelerated expansion. In order to explain current acceleration of the universe, we require an exotic energy dubbed "dark energy" with equation of state satisfying $\omega<-\frac{1}{3}$.

Many models have been proposed so far to fit these observations. Among these models, the most typical ones are cosmological constant and a time varying scalar field with positive or negative kinetic energy evolving in a specific potential, referred to as "quintessence" [4-14] or "phantom" [15-19]. The essential characteristics of these dark energy models are contained in the parameter of its equation of state, $p=\omega \rho$, where $p$ and $\rho$ denote the pressure and energy density of dark energy, respectively, and $\omega$ is a state parameter. Quintessence model has been widely studied, and its state parameter $\omega$ which is time-dependent, is greater than -1 . At the same time, observations also suggest another dark energy model - phantom with an equation of state $\omega<-1$. The most typical characteristic of the phantom model is that the kinetic of the scalar field is negative. Because of this, it leads to some unusual features of phantom model. A striking consequence of phantom model is that the Universe will undergo a catastrophic "Big Rip" in a finite time. However, Hao and Li[20] have presented that a de Sitter attractor will prevent the phantom energy from increasing up to infinite in a finite cosmic time, therefor the presence of phantom energy dose not lead to a cosmic doomsday in a theory with de Sitter attractor at late time. On the other hand, it has been shown that big rip singularity can be generically avoided if one considers quantum gravitational corrections to dynamics[21].

Some authors have considered the coupled dark energy[22]. In the work of L.Amendola[23], he investigated the cosmological consequences of the coupled quintessence model, assuming an exponential potential. In our previous paper[24], we have considered a dilatonic dark energy model, based on Weyl-scaled induced gravitational theory. In that paper, we find that when the dilaton field is not gravitational clustered at small scales, the effect of dilaton can not change the evolutionary law of baryon density perturbation, and the density perturbation can grow from $z \sim 10^{3}$ to $z \sim 5$, which guarantees the structure formation. When dilaton energy is very small compared the matter energy, potential energy of dilaton field can be neglected. In this case, the solution of cosmological scale $a$ has been found[25]. In what follows, we shall consider a more general dilatonic dark energy model that the contributions from radiation and matter can't be neglected. In this model, we will study coupled quintessence - dilaton field with positive kinetic energy, and 
phantom-dilaton field with negative kinetic energy and find out the sufficient conditions of existence of attractor solutions, which limit the choice of potential of scalar field. When we take the potential as the form $\frac{\mu}{4}\left(\sigma^{2}-\varepsilon^{2}\right)^{2}+W_{0}$ named Mexican hat potential, we investigate existence and dependence on initial conditions for a late time attractor and obtain the sufficient conditions for the existence of an attractor solutions. We find that these attractor solutions correspond to an equation of state $\omega=-1$ and a cosmic density parameter $\Omega_{\sigma}=1$, which are important features for a dark energy model that can meet the current observations. These results are shown mathematically. Finally, we show the effect of coupling term on the evolution of $X$ and $Y$ with respect to $N$ numerically.

This paper is organized as follows: In section 2, we introduce Weyl-scaled induced gravitational theory and regard dilaton as quintessence. Based on this, we investigate the sufficient condition for existence of attractor solution. In section 3, we consider phantom model and give the sufficient condition for existence of attractor solution. Section 4 is a special example-Mexican hat potential. Section 5 is summery.

\section{Coupled Quintessence}

To deduce the field equation of induced gravitational theory, let us consider the action of Jordan-BransDicke theory firstly

$$
S=\int d^{4} X \sqrt{-\gamma}\left[\phi \tilde{R}-\omega \gamma^{\mu \nu} \frac{\partial_{\mu} \phi \partial_{\nu} \phi}{\phi}-\Lambda(\phi)+\tilde{L}_{\text {fluid }}(\psi)\right]
$$

where the lagrangian density of cosmic fluid $\tilde{L}_{f l u i d}(\psi)=\frac{1}{2} \gamma^{\mu \nu} \partial_{\mu} \psi \partial_{\nu} \psi-V(\psi), \gamma$ is the determinant of $\gamma_{\mu \nu}$ which is Jordan metric, $\omega$ is the dimensionless coupling parameter, $R$ is the contracted $R_{\mu \nu}$. The metric sign convention is $(-,+,+,+)$. The quantity $\Lambda(\phi)$ is a nontrivial potential of $\phi$ field. When $\Lambda(\phi) \neq 0$ the action of Eq.(1) describes the induced gravity. The energy density of cosmic fluid $\widetilde{\rho}=\frac{1}{2}\left(\frac{d \psi}{d t}\right)^{2}+V(\psi)$ and the pressure $\widetilde{p}=\frac{1}{2}\left(\frac{d \psi}{d \vec{t}}\right)^{2}-V(\psi)$.

However it is often useful to write the action in terms of the conformally related Einstein metric. We introduce the dilaton field $\sigma$ and conformal transformation as follows

$$
\begin{gathered}
\phi=\frac{1}{2} e^{\alpha \sigma} \\
\gamma_{\mu \nu}=e^{-\alpha \sigma} g_{\mu \nu}
\end{gathered}
$$

where $\alpha^{2}=\frac{\kappa^{2}}{2 \varpi+3}$ with $\varpi>3500[26]$ being an important parameter in Weyl-scaled induced gravitational theory, $g_{\mu \nu}$ is the Pauli metric. In this paper, we work in units $\left(\kappa^{2}=8 \pi G=c=1\right)$. From the solar system tests, the current constrain is $\alpha^{2}<0.001$ [27]. The new constrain on the parameter is $\alpha^{2}<0.0001$ [28], which seems to argue against the existence of long-range scalars. Perhaps such a pessimistic interpretation of the limit is premature [27].

The action(1) becomes Eq.(4) by performing the conformal transformation Eq.(2) and Eq.(3)

$$
S=\int d^{4} X \sqrt{-g}\left[\frac{1}{2} R\left(g_{\mu \nu}\right)-\frac{1}{2} g^{\mu \nu} \partial_{\mu} \sigma \partial_{\nu} \sigma-W(\sigma)+L_{\text {fluid }}(\psi)\right]
$$

where $L_{\text {fluid }}(\psi)=\frac{1}{2} g^{\mu \nu} e^{-\alpha \sigma} \partial_{\mu} \psi \partial_{\nu} \psi-e^{-2 \alpha \sigma} V(\psi)$. The conventional Einstein gravity limit occurs as $\sigma \rightarrow 0$ for an arbitrary $\omega$ or $\omega \rightarrow \infty$ with an arbitrary $\sigma$.

The nontrivial potential of the $\sigma$ field, $W(\sigma)$ can be a metric scale form of $\Lambda(\phi)$. Otherwise, one can start from Eq.(4), and define $W(\sigma)$ as an arbitrary nontrivial potential. $g_{\mu \nu}$ is the pauli metric. Damour and Cho et.al pointed out that the pauli metric can represent the massless spin-two graviton in induced gravitational theory. Cho also pointed out that in the compactification of Kaluza-Klein theory, the physical metric must be identified as the pauli metric because of the the wrong sign of the kinetic energy term of the scalar field in the Jordan frame[29]. The dilaton field appears in string theory naturally.

By varying the action Eq.(4), one can obtain the field equations of Weyl-scaled induced gravitational theory.

$$
\begin{gathered}
R_{\mu \nu}-\frac{1}{2} g_{\mu \nu} R=-\frac{1}{3}\left\{\left[\partial_{\mu} \sigma \partial_{\nu} \sigma-\frac{1}{2} g_{\mu \nu} \partial_{\rho} \sigma \partial^{\rho} \sigma\right]-g_{\mu \nu} W(\sigma)\right. \\
\left.+e^{-\alpha \sigma}\left[\partial_{\mu} \psi \partial_{\nu} \psi-\frac{1}{2} g_{\mu \nu} \partial_{\rho} \psi \partial^{\rho} \psi\right]-g_{\mu \nu} e^{-2 \alpha \sigma} V(\psi)\right\} \\
\Delta \sigma=\frac{d W(\sigma)}{d \sigma}-\frac{\alpha}{2} e^{-2 \alpha \sigma} g^{\mu \nu} \partial_{\mu} \psi \partial_{\nu} \psi-2 \alpha e^{-2 \alpha \sigma} V(\psi) \\
\Delta \psi=-\alpha g_{\mu \nu} \partial_{\mu} \psi \partial_{\nu} \sigma+e^{-\alpha \sigma} \frac{d V(\psi)}{d \psi}
\end{gathered}
$$


The energy-momentum tensor $T_{\mu \nu}$ of cosmic fluid is

$$
T_{\mu \nu}=(\rho+p) U_{\mu} U_{\nu}+p g_{\mu \nu}
$$

where the density of energy

$$
\rho=\frac{1}{2} \dot{\psi}^{2}+e^{-\alpha \sigma} V(\psi)
$$

the pressure

$$
p=\frac{1}{2} \dot{\psi}^{2}-e^{-\alpha \sigma} V(\psi)
$$

$\rho$ and $p$ are related to their directly measurable counterparts by $\rho=e^{-\alpha \sigma} \widetilde{\rho}, p=e^{-\alpha \sigma} \widetilde{p}$.

In FRW universe, the field equations of Weyl-scaled induced gravitational theory can be expressed as follows:

$$
\begin{gathered}
H^{2}=\frac{1}{3}\left[\frac{1}{2} \dot{\sigma}^{2}+W(\sigma)+e^{-\alpha \sigma} \rho\right] \\
\ddot{\sigma}+3 H \dot{\sigma}+\frac{d W}{d \sigma}=\frac{1}{2} \alpha e^{-\alpha \sigma}(\rho-3 p) \\
\dot{\rho}+3 H(\rho+p)=\frac{1}{2} \alpha \dot{\sigma}(\rho+3 p)
\end{gathered}
$$

where $H$ is Hubble parameter. From Eq.(12), we note that there a coupling between matter and dilaton. Its effect will be discussed at the end of this section. For radiation $\rho_{r}=3 p_{r}$, we get $\rho_{r} \propto \frac{e^{\alpha \sigma}}{a^{4}}$ from Eq.(13). For matter $p_{m}=0$, we get $\rho_{m} \propto \frac{e^{\frac{1}{2} \alpha \sigma}}{a^{3}}$ from Eq.(13). Taking these results into Eqs.(11) and (12), we obtain

$$
\begin{gathered}
H^{2}=H_{i}^{2}\left[\frac{\frac{1}{2} \dot{\sigma}^{2}+W(\sigma)}{\rho_{c, i}}+\Omega_{m, i} e^{-\frac{1}{2} \alpha \sigma}\left(\frac{a_{i}}{a}\right)^{3}+\Omega_{r, i}\left(\frac{a_{i}}{a}\right)^{4}\right] \\
\ddot{\sigma}+3 H \dot{\sigma}+\frac{d W}{d \sigma}=\frac{1}{2} \alpha \eta_{i} e^{-\frac{1}{2} \alpha \sigma} a^{-3}
\end{gathered}
$$

where $H_{i}^{2}=\frac{\rho_{c, i}}{3}, \rho_{c, i}$ is the critical energy density of the universe at initial time $t_{i} . H_{i}, \Omega_{m, i}, \Omega_{r, i}$ denote the Hubble parameter, matter energy density parameter, radiation energy density parameter at initial time $t_{i}$ respectively, and $\eta_{i}=\frac{\rho_{m, i} a_{i}^{3}}{e^{\frac{1}{2} \alpha \sigma_{i}}}$ with $\rho_{m, i}$ being matter energy density at initial time $t_{i}$. We define our starting point as the equipartition epoch, at which $\Omega_{m, i}=\Omega_{r, i}=0.5$ and consider the initial scale factor $a_{i}=1$ for convenience. So we have

$$
H^{2}=H_{i}^{2}\left[\frac{\frac{1}{2} \dot{\sigma}^{2}+W(\sigma)}{\rho_{c, i}}+\Omega_{m, i} e^{-\frac{1}{2} \alpha \sigma} a^{-3}+\Omega_{r, i} a^{-4}\right]
$$

The effective density $\rho_{\sigma}$ and effective pressure $p_{\sigma}$ can be expressed as follows

$$
\begin{aligned}
& \rho_{\sigma}=\frac{1}{2} \dot{\sigma}^{2}+W(\sigma) \\
& p_{\sigma}=\frac{1}{2} \dot{\sigma}^{2}-W(\sigma)
\end{aligned}
$$

So, the equation of state of dilaton field is

$$
\omega_{\sigma}=\frac{\frac{1}{2} \dot{\sigma}^{2}-W(\sigma)}{\frac{1}{2} \dot{\sigma}^{2}+W(\sigma)}
$$

In order to gain more insights into the dynamical system, we introduce the new dimensionless variables

$$
X=\frac{\sigma}{\sigma_{0}}, \quad Y=\frac{\dot{\sigma}}{\sigma_{0}^{2}}, \quad N=\ln a
$$

Eq.(19) becomes

$$
\omega_{\sigma}=\frac{\frac{\sigma_{0}^{4} Y^{2}}{2}-W(X)}{\frac{\sigma_{0}^{4} Y^{2}}{2}+W(X)}
$$

The field Eqs.(14)(15) could be rewritten as follows

$$
\frac{d X}{d N}=\frac{\sigma_{0} Y}{H}
$$




$$
\frac{d Y}{d N}=-3 Y-\frac{W^{\prime}(X)}{\sigma_{0}^{3} H}+\frac{\alpha \eta_{i} e^{-\frac{1}{2} \alpha \sigma_{0} X} e^{-3 N}}{4 H}
$$

where the prime denotes the derivative with respect to $X$ and $H$ could be expressed as

$$
H=H_{i}\left[\frac{\frac{1}{2} \sigma_{0}^{4} Y^{2}+W(X)}{\rho_{c, i}}+\Omega_{m, i} e^{-\frac{1}{2} \alpha \sigma_{0} X} a^{-3 N}+\Omega_{r, i} a^{-4 N}\right]^{\frac{1}{2}}
$$

According to reference[30], the dynamic system decided by Eqs.(22)(23) belongs to the class of so called autonomous systems. The coordinates of critical point is decided by the following condition

$$
\frac{d X}{d N}=0, \quad \frac{d Y}{d N}=0
$$

In dilaton-dominant epoch, the matter energy density can be neglected comparing to the dilaton energy density, that is $\frac{\alpha \eta_{i} e^{-\frac{1}{2} \alpha \sigma_{0} X} e^{-3 N}}{4 H} \rightarrow 0$. So autonomous systems (22)(23) become

$$
\begin{gathered}
\frac{d X}{d N}=\frac{\sigma_{0} Y}{H} \\
\frac{d Y}{d N}=-3 Y-\frac{W^{\prime}(X)}{\sigma_{0}^{3} H}
\end{gathered}
$$

The critical point of the above autonomous system is $\left(X_{c}, 0\right)$, where $X_{c}$ is defined by $W^{\prime}\left(X_{c}\right)=0$. Linearize the above equations about the critical point, we have

$$
\begin{gathered}
\frac{d X}{d N}=\frac{\sqrt{3} \sigma_{0} Y}{\sqrt{W\left(X_{c}\right)}} \\
\frac{d Y}{d N}=-3 Y-\frac{\sqrt{3} W^{\prime \prime}\left(X_{c}\right) X}{\sigma_{0}^{3} \sqrt{W\left(X_{c}\right)}}
\end{gathered}
$$

The eigenvalues of the system are

$$
x_{1,2}=\frac{-\xi \pm \sqrt{\xi^{2}-4 \zeta}}{2}
$$

where $\xi=3$ and $\zeta=\frac{W^{\prime \prime}\left(X_{c}\right)}{W\left(X_{c}\right) \sigma_{0}^{2}}$. For a positive potential, if $W^{\prime \prime}\left(X_{c}\right)>0$, i.e. $x_{1}<0$ and $x_{2}<0$, the critical point is a stable point which corresponds to a late time attractor solution. So, $W\left(X_{c}\right)>0, W^{\prime}\left(X_{c}\right)=0$ and $W^{\prime \prime}\left(X_{c}\right)>0$ is the sufficient condition for existence of attractor solution in quintessence model. In this paper we take potential $W(X)$ as the Mexican hat potential which satisfies the above condition $W\left(X_{c}\right)>0$, $W^{\prime}\left(X_{c}\right)=0$ and $W^{\prime \prime}\left(X_{c}\right)>0$. Therefore, our quintessence model can admit a late time attractor solution.

Based on the analysis of the above autonomous system, we can conclude that: Because $\frac{\alpha \eta_{i} e^{-\frac{1}{2} \alpha \sigma_{0} X} e^{-3 N}}{4 H} \rightarrow$ 0 , the coupling between matter and dilaton can't change existence of attractor solution in Eqs.(22)(23), that is to say, the coupled term can't change the fate of the evolution of the universe. In one word, the existence of couple term $\frac{\alpha \eta_{i} e^{-\frac{1}{2} \alpha \sigma_{0} X} e^{-3 N}}{4 H}$ between matter and dilaton affects the evolutive process of the universe, but not the fate of the universe.

In what follows, we shall show mathematically the evolutions of the components of cosmic density $\Omega$, the evolution of the parameter of state equation $\omega$ and the evolution of $X, Y$ with respect to $N$ in quintessence model. The attractor behavior is also shown in phase plane.

\section{Phantom Model}

When we regard dilaton as phantom field, its kinetic energy will be negative. So, the phantom field equations become

$$
\begin{gathered}
H_{p}^{2}=\frac{1}{3}\left[-\frac{1}{2} \dot{\sigma}^{2}+W(\sigma)+e^{-\alpha \sigma} \rho\right] \\
\ddot{\sigma}+3 H_{p} \dot{\sigma}-\frac{d W}{d \sigma}=\frac{1}{2} \alpha e^{-\alpha \sigma}(\rho-3 p)
\end{gathered}
$$

Based on the same analysis like the quintessence model, we have,

$$
H^{2}=H_{i}^{2}\left[\frac{-\frac{1}{2} \dot{\sigma}^{2}+W(\sigma)}{\rho_{c, i}}+\Omega_{m, i} e^{-\frac{1}{2} \alpha \sigma}\left(\frac{a_{i}}{a}\right)^{3}+\Omega_{r, i}\left(\frac{a_{i}}{a}\right)^{4}\right]
$$




$$
\ddot{\sigma}+3 H \dot{\sigma}-\frac{d W}{d \sigma}=\frac{1}{2} \alpha \eta_{i} e^{-\frac{1}{2} \alpha \sigma} a^{-3}
$$

According to the transition (20), the phantom equation fields become

$$
\begin{gathered}
\frac{d X}{d N}=\frac{\sigma_{0} Y}{H} \\
\frac{d Y}{d N}=-3 Y+\frac{W^{\prime}(X)}{\sigma_{0}^{3} H}+\frac{\alpha \eta_{i} e^{-\frac{1}{2} \alpha \sigma_{0} X} e^{-3 N}}{4 H}
\end{gathered}
$$

where the prime denotes the derivative with respect to $X$, and $H$ can be expressed as

$$
H=H_{i}\left[\frac{-\frac{1}{2} \sigma_{0}^{4} Y^{2}+W(X)}{\rho_{c, i}}+\Omega_{m, i} e^{-\frac{1}{2} \alpha \sigma_{0} X} a^{-3 N}+\Omega_{r, i} a^{-4 N}\right]^{\frac{1}{2}}
$$

The equation of state parameter becomes

$$
\omega_{\sigma}=\frac{-\frac{\sigma_{0}^{4} Y^{2}}{2}-W(X)}{-\frac{\sigma_{0}^{4} Y^{2}}{2}+W(X)}
$$

Eqs.(35)(36) and their initial conditions determine the evolution of phantom universe and the behaviors of the late time de Sitter attractor, which include the existence and the stability of the solution. According to the similar analysis to the quintessence model, we also conclude: $W\left(X_{c}\right)>0, W^{\prime}\left(X_{c}\right)=0$ and $W^{\prime \prime}\left(X_{c}\right)<$ $0\left(x_{1}<0, x_{2}<0\right)$ is the sufficient condition for existence of attractor solution in phantom model. In this paper we take potential $W(X)$ as the Mexican hat potential which satisfies the above condition $W\left(X_{c}\right)>0$, $W^{\prime}\left(X_{c}\right)=0$ and $W^{\prime \prime}\left(X_{c}\right)<0$. Therefore, our phantom model can admit a late time attractor solution.

In what follows, we shall show mathematically the evolutions of the components of cosmic density $\Omega$, the evolution of the parameter of state equation $\omega$ and the evolution of $X, Y$ with respect to $N$ in phantom model. The attractor behavior is also shown in phase plane.

\section{Mexican Hat Potential $\frac{\mu}{4}\left(\sigma^{2}-\varepsilon^{2}\right)^{2}+W_{0}$}

For this type of Mexican hat potential, it has two extremum points in the range $\sigma \geq 0$ : a minimum at $\sigma=\varepsilon$ and a maximum at $\sigma=0$. The non-conventional parameter $W_{0}$ in this potential, moves the potential up and down, which is equivalent to adding a cosmological constant to the usual Mexican hat potential. We show the feature of Mexican hat potential mathematically in Fig.1.

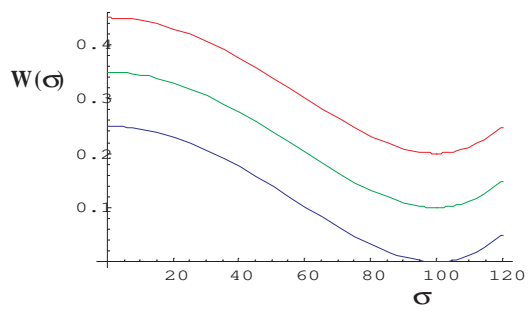

Fig.1 Mexican hat potential when we set $W_{0}=0($ lower line $), 0.1$ (middle line), 0.2(upper line).

The critical point of the above autonomous system is $\left(X_{c}, 0\right)$, where $X_{c}$ is defined by $W^{\prime}\left(X_{c}\right)=0$. Note that the energy density of the dilaton field at the critical point is $W\left(X_{c}\right)$ and should not vanish, thus the sufficient condition for the existence of a viable cosmological model with a late time de Sitter attractor solution should be that: the potential of the field has non-vanishing minimum value in quintessence model, on the contrary, the potential should have non-vanishing maximum value in phantom model. The extremum difference of potential between quintessence model and phantom model results from the unusual physical feature of phantom field.

Since the Mexican hat potential $\left(W_{0} \neq 0\right)$ has a non-vanishing minimum value and a non-vanishing maximum value, there must exist late time attractors in the quintessence model and phantom model, which both drive from dilaton field. Based on this, we study the attractor behaviors of quintessence and phantom model in Mexican hat potential.

In quintessence model, the critical point is $X_{c 1}=\varepsilon$, and at this point the potential has a minimum value $W_{0}$. Obviously, it is an attractor solution, which corresponds to an equation of state parameter $\omega=-1$ and a cosmic density parameter $\Omega_{\sigma}=1$. The evolution of the parameter of state equation $\omega$, the cosmic density 
parameter $\Omega, Y$ with respect to $N$ are shown numerically in Figs.2-4. We also show the attractor property for quintessence in phase plane in Fig.5.

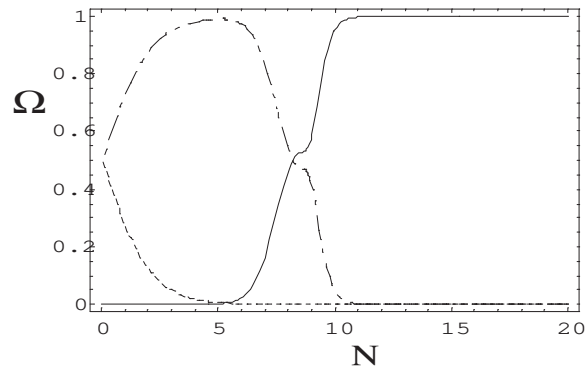

Fig.2 The evolution of cosmic density parameters $\Omega_{\sigma}$ (solid line $), \Omega_{m}($ dash - dot line $)$, $\Omega_{r}($ dot line $)$ with respect to $\mathrm{N}$ in Mexican hat potential for quintessence. We set $\frac{\mu}{H_{i}}=10^{-12}$, $\alpha=0.01, W_{0}=1.00$.

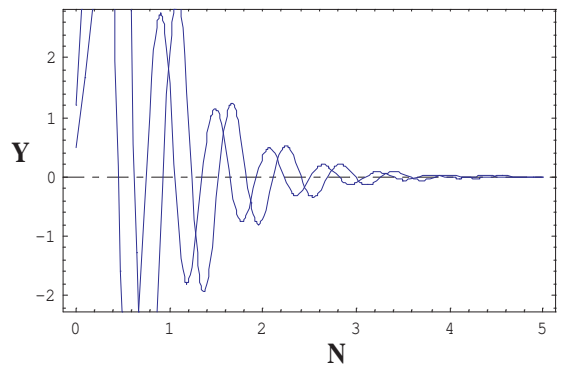

Fig.4

The evolution of $\mathrm{Y}$ with respect to $\mathrm{N}$ for different coordinate initial conditions $X_{i}=0.5$, $Y_{i}=0.5 ; X_{i}=1.2, Y_{i}=1.2$ in Mexican hat potential for quintessence.

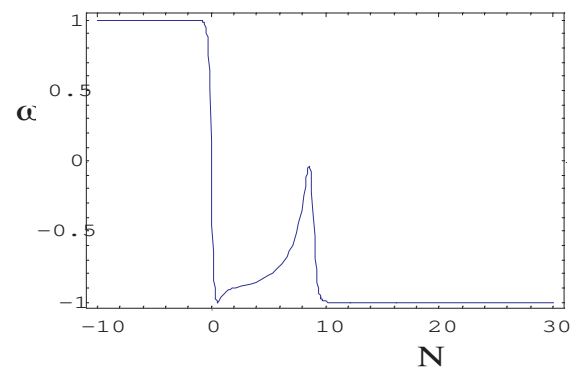

Fig.3

The evolution of the parameter of state equation $\omega$ with respect to $\mathrm{N}$ in Mexican hat potential for quintessence. We set $\frac{\mu}{H_{i}}=10^{-12}$, $\alpha=0.01, W_{0}=1.00$.

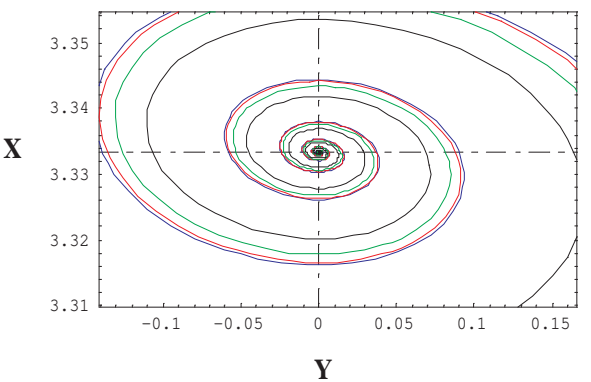

Fig.5 The phase portrait for quintessence in Mexican hat potential for several different initial conditions. The critical point $X_{c}=3.333$ corresponds to a de Sitter solution which is a stable spiral

In phantom model, according to the sufficient condition of existence of attractor solution, we can obtain the critical point is $X_{c 2}=0$, and at this point the potential has a maximum value $\frac{\mu \varepsilon^{4}}{4}+W_{0}$. Obviously, it is also an attractor solution, which corresponds to an equation of state parameter $\omega=-1$ and a cosmic density parameter $\Omega_{\sigma}=1$. The evolution of the parameter of state equation $\omega$ and the cosmic density parameter $\Omega$ with respect to $N$ are shown numerically in Fig.6 and Fig.7. We also show the attractor property for phantom in phase plane in Fig.8.

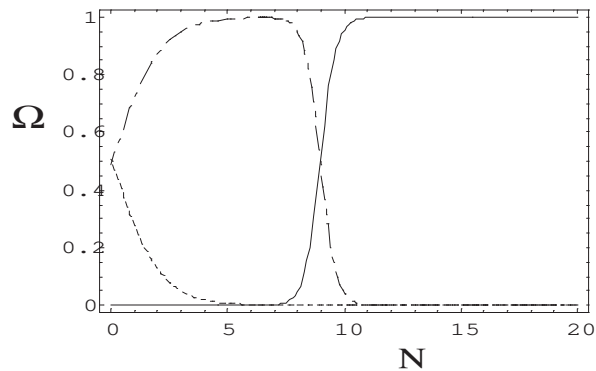

Fig.6 The evolution of cosmic density parameters $\Omega_{\sigma}$ (solid line), $\Omega_{m}($ dash - dot line), $\Omega_{r}($ dot line $)$ with respect to $\mathrm{N}$ in Mexican hat potential for phantom. We set $\frac{\mu}{H_{i}}=10^{-15}$, $\alpha=0.01, W_{0}=1.00$.

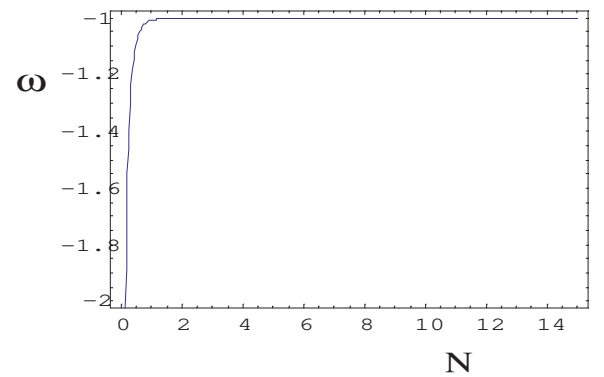

Fig.7 The evolution of the parameter of state equation $\omega$ with respect to $\mathrm{N}$ in Mexican hat potential for phantom. We set $\frac{\mu}{H_{i}}=10^{-15}$, $\alpha=0.01, W_{0}=1.00$. 


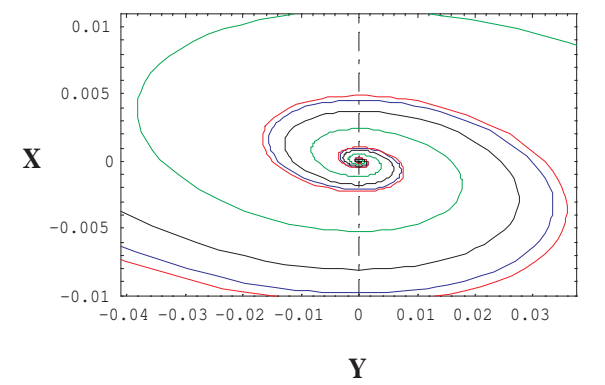

Fig. 8 The phase portrait for phantom in Mexican hat potential for several different initial conditions. The critical point $X_{c}=0$ corresponds to a de Sitter solution which is a stable spiral

In what follows, we shall show the effect of nonminimum coupling term $\frac{\alpha \eta_{i} e^{-\frac{1}{2} \alpha \sigma_{0} X} e^{-3 N}}{4 H}$ on the evolution of $X, Y$ and the attractor solution. From Figs.9-11 and the analysis in Section 2, we obtain that the existence of couple term between matter and dilaton affects the evolution course of the universe but not the result. That is to say, either quintessence or phantom model admits an attractor in spite of the existence of coupling term.

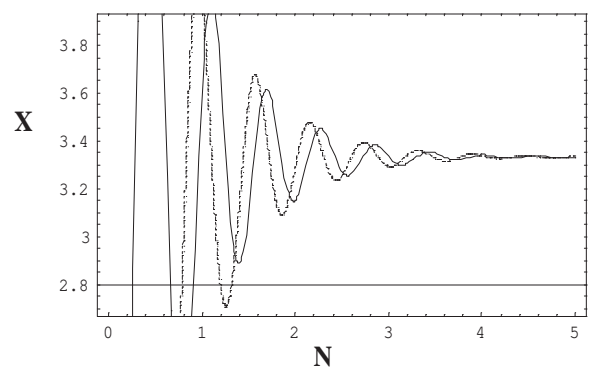

Fig.9 The evolutive comparison of $X$ with respect to $N$ in quintessence with coupling term(real line) and without coupling term(dot line).

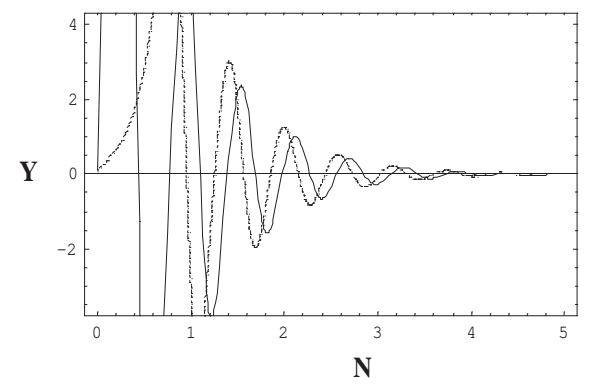

Fig.10 The evolutive comparison of $Y$ with respect to $N$ in quintessence with coupling term(real line) and without coupling term(dot line).

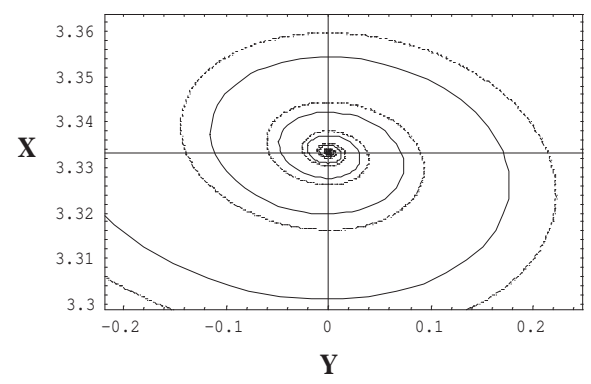

Fig.11 The phase portrait comparison for quintessence with coupling term(real line) and without coupling term. Both of them admit the same one attractor $X_{c}=3.33$.

\section{Conclusions}

In this paper, we consider dilaton field as quintessence and phantom in dilatonic dark energy model. We investigate the existence of a late time attractor in the the two cases. The sufficient condition of the existence of attractor solution is: the potential of the field has non-vanishing minimum value in quintessence model, on the contrary, the potential should have non-vanishing maximum value in phantom model. The physical reason of the condition difference between quintessence and phantom is clarified as follows: For a usual dynamic system(quintessence), the system will be stable when the total energy of the system arrives at the minimum value. So, for quintessence the sufficient condition is that the potential of the field has non-vanishing minimum value. However, for a phantom system whose kinetic energy is negative, the system will be stable when the total energy of the system arrives at the maximum value. So, for phantom the sufficient condition is that the potential of the field has non-vanishing maximum value.

The Mexican hat potential has a local minimum and a local maximum which makes us consider quintessence and phantom in the same potential. In quintessence model, the critical point is $X_{c 1}=\varepsilon$, and at this point 
the potential has a minimum value $W_{0}$. In phantom model, we obtain that the critical point is $X_{c 2}=0$, and at this point the potential has a maximum value $\frac{\mu \varepsilon^{4}}{4}+W_{0}$. We show mathematically that both quintessence model and phantom model admits a late time attractor solution corresponding to an equation of state $\omega=-1$ (Fig.3 and Fig.7) and a cosmic density parameter $\Omega_{\sigma}=1$ (Fig.2 and Fig.6), which are important features for a dark energy model that can meet the current observations. Such evolution behaviors will avoid the cosmic doomsday. Since $T_{e q} \simeq 5.64\left(\Omega_{0} h^{2}\right) e V \simeq 2.843 \times 10^{4} K, T_{0} \simeq 2.7 K, a_{i}=1$, the scale factor at the present epoch $a_{0}$ would nearly be $1.053 \times 10^{4}$, then we know $N_{0}=\ln a_{0}=9.262$. According to $N_{0}$, we obtain the current value $\Omega_{\sigma, q u i n t e s s e n c e} \simeq 0.707036$ and $\Omega_{\sigma, \text { phantom }} \simeq 0.703174$, which both meet the current observations well.

Finally, we analyze the effect of the coupling term $\frac{\alpha \eta_{i} e^{-\frac{1}{2} \alpha \sigma_{0} X} e^{-3 N}}{4 H}$ on the evolution of $X, Y$ and attractor(Figs.9-11). We find that it changes the evolutive process of $X$ and $Y$, but not the existence of the attractor solution. These mathematical results show that, because of the existence of this coupling between dilaton and matter, the evolution of $X$ and $Y$ with respect to $N$ in quintessence model will be faster than those of the other models without coupling. According to the same analysis methods, we can expect that the coupling term affects the evolution of $\omega$ and $\Omega$ in the same way.

We can find that the state parameter of dilaton as quintessence and phantom can both evolve to -1 from values greater than -1 and from values smaller than -1 , respectively. Can it cross over -1 ? Or dilaton can be a candidate of quintom[31]? Vikman has proved that the crossing of $\omega=-1$ is classically forbidden in the single scalar field models[32]. However, in the work of Singh [33], we can observe such a behavior of crossing of $w=-1$ in semi-classical Loop Quantum Cosmology very naturally. If one considers the quantum effects, such a crossing is possible for a single scalar field.

\section{Acknowledgements}

This work is partially supported by National Nature Science Foundation of China under Grant No.10573012 and Shanghai Municipal Science and Technology Commission No.04dz05905.

\section{References}

1. A. G. Riess et al., Astrophys. J607, 665(2004);

A. G. Riess, Astron. J116, 1009(1998);

S. Perlmutter et al., Astrophys. J517, 565(1999);

N. A Bahcall et al., Science 284, 1481(1999).

2. C. L. Bennett et al., Astrophys. J. Lett148, 1(2003).

3. M. Tegmark et al., Phys. Rev. D69, 103510(2004);

M. Tegmark et al., Astrophys. J606, 702(2004).

4. J. S. Bagla, H. K. Jassal and T. Padmamabhan, Phys. Rev. D67, 063504(2003).

5. E. Elizalde, S. Nojiri and S. D. Odintsov, hep-th/0405034

S. Nojiri, S. D. Odintsov and M. Sasaki, hep-th0504052

S. Nojiri and S. D. Odintsov, hep-th/0601213

6. C. Wetterich Nucl. Phys. B302, 668(1998);

P. G. Ferreira and M. Joyce Phys. Rev. D58, 023503(1998);

J. Frieman, C. T. Hill, A. Stebbinsand and I.Waga, Phys. Rev. Lett75, 2077(1995);

P. Brax and J. Martin, Phys. Rev. D61, 103502(2000)

T. Barreiro, E. J. Copeland and N. J. Nunes, Phys. Rev. D61, 127301(2000);

I. Zlatev, L. Wang and P. J. Steinhardt Phys. Rev. Lett 82, 896(1999).

7. T. Padmanabhan, and T. R. Choudhury, Phys. Rev. D66, 081301(2002).

8. A. Sen, JHEP 0204, 048(2002).

9. C. Armendariz-Picon, T. Damour and V. Mukhanov, Phys. Lett. B458, 209(1999).

10.A. Feinstein, Phys. Rev. D66, 063511(2002);

M. Fairbairn and M. H. Tytgat, arXiv:hep-th/0204070

G. W. Gibbons, arXiv:hep-th/0204008

11.A. Frolov, L. Kofman and A. Starobinsky, Phys.Lett.B 545, 8(2002);

L. Kofman and A. Linde, JHEP0207, 004(2004).

12.C. Acatrinei and C. Sochichiu, Mod. Phys. Lett. A18, 31(2003);

S. H. Alexander, Phys. Rev. D65, 0203507(2002).

13.T. Padmanabhan, Phys. Rev. D66, 021301(2002).

14.A. Mazumadar, S. Panda and A. Perez-Lorenzana, Nucl. Phys. B614, 101(2001);

S. Sarangi and S. H. Tye, Phys. Lett. B536, 185(2002). 
15.H. Q. Lu, Int. J. Mod. Phys. D14, 355(2005);

W. Fang, H. Q. Lu, Z. G. Huang and K. F. Zhang, Int. J. Mod. Phys. D15, 199(2006).

16.X. Z. Li and J. G. Hao, Phys. Rev. D69, 107303(2004).

17.T. Chiba, T. Okabe and M. Yamaguchi, Phys. Rev. D62, 023511(2002).

18.P. Singh, M. Sami and N. Dadhich, arXiv:hep-th/0305110

19.S. M. Carroll, M. Hoddman and M. Trodden, arXiv:astro-ph/0301273

20.J. G. Hao and X. Z. Li, Phys. Rev. D68, 083514(2003).

21.M. Sami, P. Singh, and S. Tsujikawa, arXiv:gr-qc/0605113

22.Michael Doran and J. Jächel, Phys. Rev. D66, 043519(2002);

N. J. Nunes and D. F. Mota, arXiv:astro-ph/0409481 and astro-ph/0504519

Ishwaree P. Neupane, arXiv:hep-th/0602097

Benedict M.N. Carter and Ishwaree P. Neupane, arXiv:hep-th/0512262 and hep-th/0510109

Federico Piazza and Shinji Tsujikawa, arXiv:hep-th/0405054

B. Gumjudpai, T. Naskar, M. Sami, and S. Tsujikawa, arXiv:hep-th/0502191

23.L. Amendola, Phys. Rev. D62, 043511(2000);

E. Majerotto, D. Sapone and L. Amendola, arXiv:astro-ph/0410543

24.H. Q. Lu, Z. G. Huang, W. Fang and K. F. Zhang, arXiv:hep-th/0409309

25.H. Q. Lu and K. S. Cheng, Astrophysics and Space Science235, 207(1996);

Y. G. Gong, arXiv:gr-qc/9809015

26.C. M. Will, arXiv:gr-qc/0103036

27.T. Damour and K. Nordtvedt, Phys. Rev. Lett70, 2217(1993).

28.B. Bertotti, L. Iess and P. Tortora, Nature425, 374(2003).

29.Y .M .Cho, Phys. Rev.Lett68, 3133(1992).

30.E. J. Copeland, M. Sami and S. Tsujikawa, arXiv:hep-th/0603057

31.W. Hao, R. G. Cai and D. F. Zeng, Class.Quant.Grav22, 3189(2005);

Z. K. Guo, Y. S. Piao, X. M. Zhang, Y.Z. Zhang, Phys.Lett. B608, 177(2005);

B. Feng, arXiv:astro-ph/0602156

32.A. Vikman, Phys. Rev. D71, 023515(2005).

33.P. Singh, Class. Quant. Grav.22, 4203(2005). 